\title{
Development of Epidermoid Metaplasia of the Mucosa in Association with Esophageal Intramural Pseudodiverticulosis and Candidiasis
}

\author{
Masako Shintaku $^{a} \quad$ Masayuki Shintaku $^{b} \quad$ Ikuko Torii $^{c}$ \\ aDepartment of Gastroenterology, Japan Community Health care Organization, Hoshigaoka \\ Medical Center, Hirakata, Japan; ${ }^{b}$ Department of Pathology, Hikone Municipal Hospital, \\ Hikone, Japan; 'Department of Diagnostic Pathology, Japan Community Health care \\ Organization, Hoshigaoka Medical Center, Hirakata, Japan
}

Keywords

Esophagus · Intramural pseudodiverticulosis · Epidermoid metaplasia · Candidiasis

\begin{abstract}
We report a case of epidermoid metaplasia of the esophageal mucosa that developed in a patient with a long history of esophageal intramural pseudodiverticulosis (EIPD) complicated by candidiasis. The patient, a 69-year-old man, had been treated for about 3 years for EIPD with candidiasis. After candidiasis improved, the development of epidermoid metaplasia of the esophageal mucosa was observed. It comprised longitudinally arranged, multiple, small, whitish flecks with a scaly appearance on the mucosa of the middle to lower esophagus, and pathological examination demonstrated several fine keratohyalin granules in superficial layers of the squamous epithelium. Six months later, candidiasis was almost cured, but these small flecks had slightly increased in size, and pathological examination demonstrated epidermoid metaplasia consisting of a thick, acellular keratin layer and well-developed granular layer beneath it. We considered that chronic candida esophagitis played the principal pathogenetic role in the development of epidermoid metaplasia. EIPD may have provided an environment suitable for the growth of fungi, and mucinous material contaminated by Candida and excreted from the orifices of EIPD may have irritated the mucosa and induced epidermoid metaplasia.
\end{abstract}


Fig. 1. Endoscopic view of the esophagus. Many small mucosal holes representing the orifices of EIPD were scattered, and several white coatings indicating Candida infection were observed. EIPD, esophageal intramural pseudodiverticulosis.

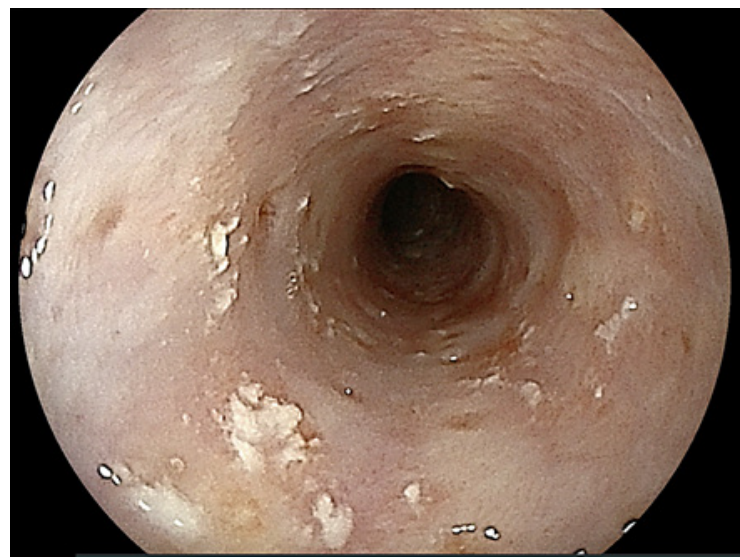

\section{Introduction}

Esophageal intramural pseudodiverticulosis (EIPD) is a rare disorder characterized by the presence of multiple, small, flask-shaped outpouchings within the esophageal wall that have orifices opening onto the esophageal mucosa [1-9]. These outpouchings are formed by dilatation of the terminal excretory ducts of esophageal submucosal mucous glands (pseudodiverticula) $[2-4,6,7]$, and we previously reported that their orifices periodically repeated opening and closing movements and excreted mucinous secretions onto the mucosal surface [8]. Thus, EIPD is not a static condition, but rather an active, continuously moving pathological process.

During a long-term endoscopic observation of a patient with EIPD complicated by esophageal candidiasis, we observed that the esophageal mucosa along the orifices of EIPD developed multifocal, small, whitish flecks corresponding to "epidermoid metaplasia" (or "epidermization") [10-13]. This report presents the endoscopic and pathological findings of this rare mucosal change of the esophagus.

\section{Case Report}

The patient, a 69-year-old male office worker, was incidentally found to have esophageal candidiasis at an annual medical checkup and referred to our hospital 3 years previously. He was an ex-smoker and had a history of moderate alcohol intake. He complained of neither dysphagia nor any other gastrointestinal symptoms. Radiological and endoscopic examinations demonstrated diffuse EIPD involving almost the entire length of the esophagus (Fig. 1), esophageal candidiasis, mild GERD, and atrophic gastritis. The orifices of EIPD periodically repeated active opening and closing movements and excreted turbid mucinous material onto the mucosal surface. The esophageal lumen showed mild narrowing, especially in the upper and lower portions, most likely due to submucosal chronic inflammation and fibrosis. Administration of antifungal drugs and proton pump inhibitors was started, and antibiotics for eradication of Helicobacter pylori were also given.

Thereafter, the patient was followed by periodic endoscopic examinations at 3- or 6 -month intervals, and candidiasis showed repeated relapses and remissions for about 2 years. When candidiasis relapsed, movements of the orifices of EIPD tended to be reduced, and some of the orifices were covered by white coatings that represented exudates produced by Candida infection. The esophageal mucosa showed diffuse and mild redness and edema. The patient remained almost asymptomatic throughout the clinical course. 

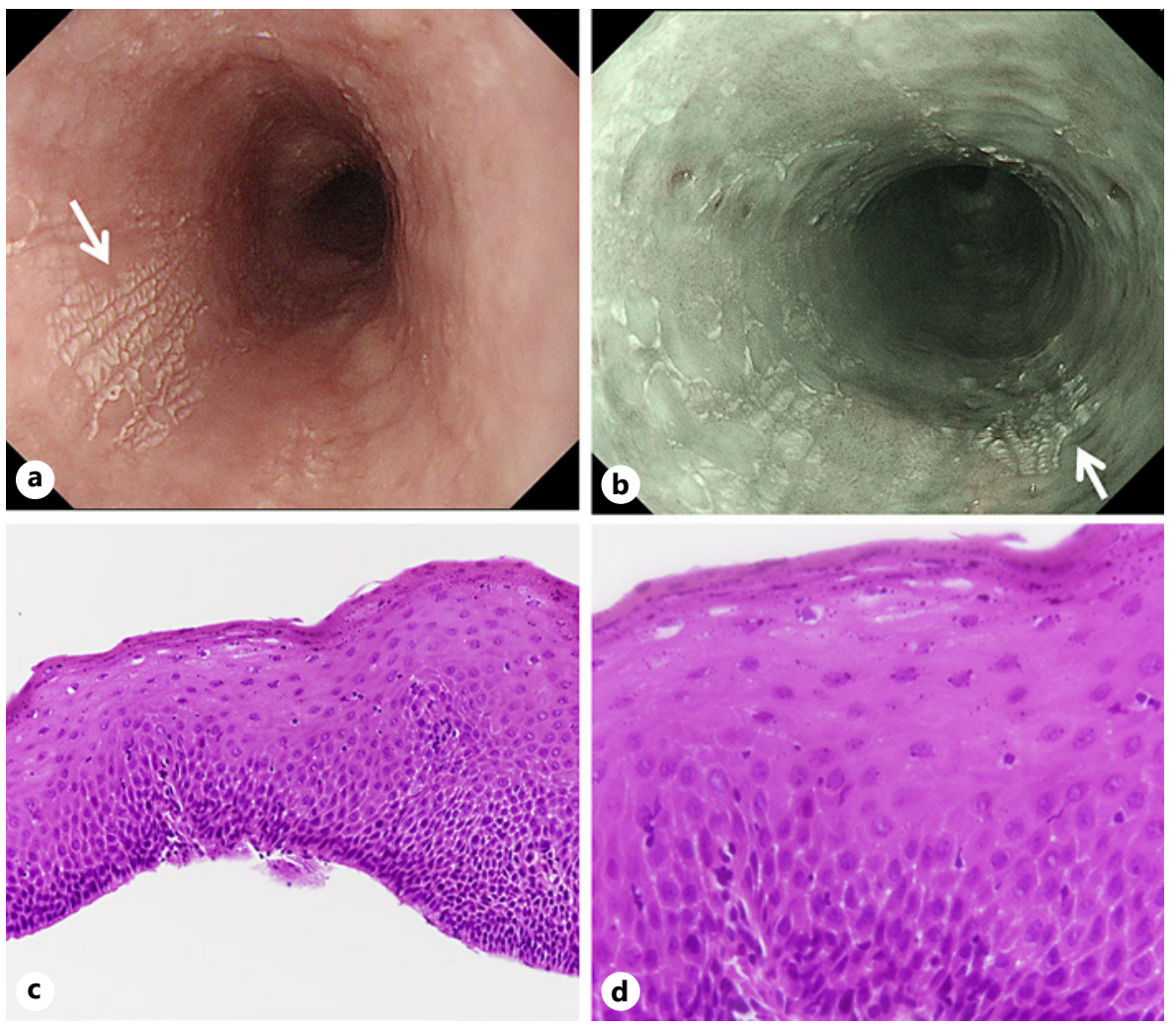

Fig. 2. a, b Endoscopic views of the esophagus. Multiple, small, whitish, scaly flecks (arrows) were longitudinally aligned from the middle to lower esophagus. White light (a). Narrow band imaging (b). Esophageal biopsy specimen $(\mathbf{c}, \mathbf{d})$. Several fine keratohyalin granules were seen in the superficial layers of the acanthotic epithelium. No hyperkeratosis was noted in this specimen (hematoxylin-eosin stain, $\mathbf{c} \times 200, \mathbf{d} \times 400$ ).

On endoscopy performed 2 and a half years after the first visit, multiple, small, whitish, scaly flecks were noted on the mucosal surface (Fig. 2a, b). They were longitudinally aligned and mainly found along the orifices of EIPD from the middle to lower esophagus. A biopsy specimen taken from one of these flecks showed mild acanthosis, and epithelial cells of the superficial layers had several fine keratohyalin granules in the cytoplasm (Fig. 2c, d). No fungal pseudohyphae of Candida species were detected.

On endoscopy performed 6 months later, whereas candidiasis was almost cured, multiple, small, whitish flecks slightly increased in size (Fig. 3a, b). Biopsy specimens taken from the flecks demonstrated a thick, acellular keratin layer with a basket-weave appearance atop the epithelium showing acanthosis, and a well-developed granular layer with abundant keratohyalin granules was noted beneath the keratin layer (Fig. 3c, d). The epithelial cells did not exhibit cellular atypia, and no formation of ectopic sebaceous glands was noted. The lamina propria was not included in the biopsy specimens, and no inflammatory cell infiltration was seen within the squamous epithelium. No fungal pseudohyphae were observed.

\section{Discussion}

Epidermoid metaplasia (or "epidermization") is a rare pathological alteration of the esophageal mucosa that is endoscopically characterized by well-demarcated areas showing a whitish, scaly, or "cobblestone-like" appearance $[11,13]$. It is clearly visualized as an 


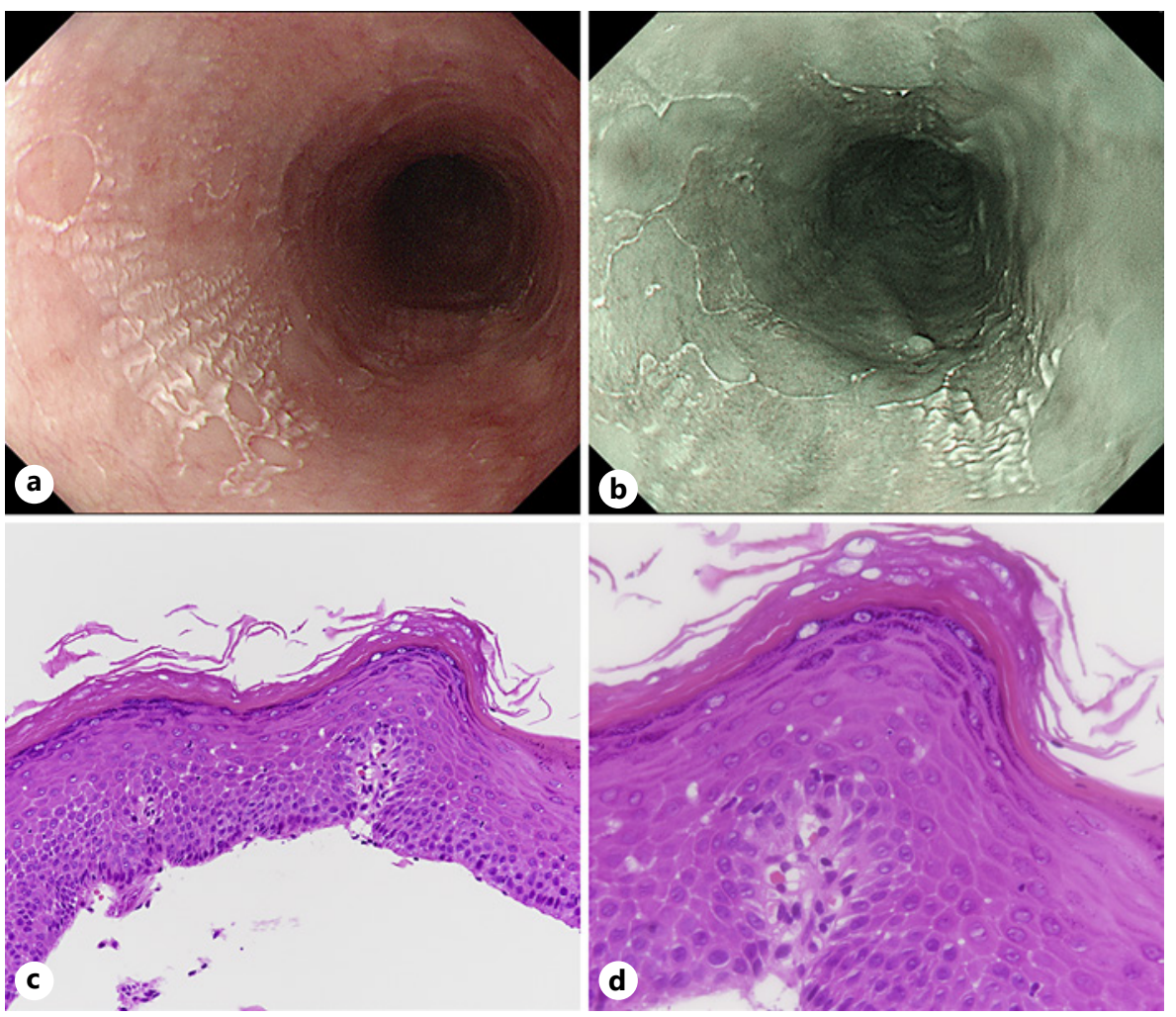

Fig. 3. a, b Endoscopic views of the same regions taken 6 months after Fig. 2. The whitish, scaly flecks slightly increased in size. White light (a). Narrow band imaging (b). Esophageal biopsy specimen (c, d). The epithelium showed marked hyperkeratosis, and a prominent granular layer was formed beneath the acellular keratin layer (hematoxylin-eosin stain, $\mathbf{c} \times 200$, $\mathbf{d} \times 400$ ).

irregular area that does not stain with Lugol's iodine method $[10,11]$. On endoscopy, it should be differentiated from localized esophagitis, glycogenic acanthosis, candidiasis, and, most importantly, superficial squamous cell carcinoma, especially carcinoma of the depressed superficial type (type 0 -IIc) which is covered by a hyperkeratotic layer [11].

The pathological findings of epidermoid metaplasia were initially described by Nakanishi et al. [10], who demonstrated that this lesion showed an immunohistochemical expression pattern of keratin subtypes similar to that of the epidermis. In recent pathological reports of large series, Taggart et al. [12] identified 37 cases (2.0\%) of this lesion, which they termed "hyperkeratosis," on reviewing 1,845 esophageal biopsies. Singhi et al. [13] reported detailed endoscopic and pathological features of this lesion in 18 patients. Epidermoid metaplasia is pathologically characterized by the formation of a thick, hyperorthokeratotic layer atop the epithelium and a well-developed granular layer beneath it [10-13]. Whereas ectopic sebaceous glands occasionally arise in the esophageal mucosa [14], only a single case of the occurrence of sebaceous glands in association with epidermoid metaplasia has been reported [12].

The etiology and pathogenesis of epidermoid metaplasia of the esophageal mucosa remain unknown. Heavy smoking, excessive alcohol intake, and gastroesophageal reflux disease have been proposed as risk factors $[12,13]$, and some kinds of chronic inflammatory irritation are considered to play a pathogenetic role [11]. The relationship between epidermoid metaplasia and esophageal neoplasms also remains uncertain. Taggart et al. [12] divided the lesion into 2 groups: group 1 occurring in the region of Barrett esophagus 
and/or adenocarcinoma and group 2 occurring outside it, and they found that only the latter group was frequently associated with squamous cell carcinoma (in 47\%) or dysplasia (in 19\%), whereas the former group was not. Singhi et al. [13] also reported the presence of squamous cell carcinoma and/or high-grade dysplasia in adjacent areas of epidermoid metaplasia in $17 \%$ of their patients and hypothesized that epidermoid metaplasia is a genomically unstable condition and may be a precursor lesion of dysplasia or carcinoma. Therefore, it is warranted to carefully follow up patients with this lesion by repeated endoscopic examinations, although there is still no direct evidence for epidermoid metaplasia being a precancerous lesion $[11,13]$.

The present patient had a long history of EIPD. EIPD is a rare disorder, and the association of epidermoid metaplasia with EIPD has only been reported by Hentschel and Lüth [9], who identified 2 cases of epidermoid metaplasia among the 21 cases of EIPD they collected. Unfortunately, neither endoscopic nor pathological findings of these 2 cases were presented in the figures. The orifices of esophageal submucosal glands and EIPD are arranged along the longitudinal axis of the esophagus [7, 9, 15]. The longitudinal alignment of small flecks of epidermoid metaplasia in the present case suggests that epidermoid metaplasia had a close relationship with EIPD.

In the present case, endoscopic examination performed 6 months prior to the first detection of small, whitish flecks did not disclose similar changes. This indicates that metaplasia developed within a relatively short period. On the initial biopsy, the lesion consisted of several fine keratohyalin granules in the superficial layers of the epithelium, the findings probably representing an early stage of keratinization, but the formation of a thick keratin layer was not observed. The absence of mature keratinization in the initial biopsy specimen is probably due to a sampling problem, since the endoscopic appearance did not significantly differ from that at the time of the second biopsy performed 6 months later. The early morphogenetic stages of epidermoid metaplasia of the esophageal epithelium were not described in previous articles, and the findings we obtained give some suggestions regarding its pathogenetic process.

In addition to EIPD, the present patient had a long history of esophageal candidiasis. Taggart et al. [12] reported the occurrence of esophageal candidiasis in association with epidermoid metaplasia in $22 \%$ of their group 2 patients, and it may be reasonable to consider that long-standing candidiasis played principal roles in the pathogenesis of epidermoid metaplasia in the present case. Esophageal candidiasis is one of the common complications of EIPD $[5,7,9]$ probably because many small outpouchings in EIPD provide an environment suitable for colonization by and growth of fungi [5]. As we demonstrated in the previous article, the orifices of EIPD repeat active opening and closing movements and continuously excrete mucinous material onto the mucosal surface [8]. If the infectious processes, such as candidiasis, complicated EIPD, the contaminated mucinous material would be excreted or overflow from the orifices and continuously irritate the mucosal epithelial cells. It is therefore reasonable to consider that the non-keratinizing squamous epithelium of the esophageal mucosa transforms into the epithelium of the epidermal phenotype, which is more resistant to noxious stimuli such as those caused by Candida infection.

\section{Statement of Ethics}

This study protocol was reviewed and the need for approval was waived by the Ethical Review Board of Japan Community Health care Organization Hoshigaoka Medical Center. Written informed consent was obtained from the patient for publication of this case report and any accompanying images.

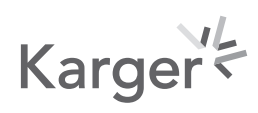


Shintaku et al.: Epidermoid Metaplasia in Esophageal Pseudodiverticulosis

\section{Conflict of Interest Statement}

The authors have no conflicts of interest to declare.

\section{Funding Sources}

The authors declare that there were no funding sources for this manuscript.

\section{Author Contributions}

Masako Shintaku performed the endoscopic examinations and drafted the manuscript and figures. Masayuki Shintaku reviewed the pathological specimens and supervised the manuscript. Ikuko Torii conducted the pathological examination.

\section{References}

1 Mendl K, McKay JM, Tanner CH. Intramural diverticulosis of the oesophagus and Rokitansky-Aschoff sinuses in the gall-bladder. BJR. 1960 Aug;33(392):496-501.

2 Boyd RM, Bogoch A, Greig JH, Trites AE. Esophageal intramural pseudodiverticulosis. Radiology. 1974 Nov; 113(2):267-70.

3 Wightman AJA, Wright EA. Intramural oesophageal diverticulosis. A correlation of radiological and pathological findings. Br J Radiol. 1974 Aug;47(560):496-8.

4 Umlas J, Sakhuja R. The pathology of esophageal intramural pseudodiverticulosis. Am J Clin Pathol. 1976 Mar; 65(3):314-20.

5 Castillo S, Aburashed A, Kimmelman J, Alexander LC. Diffuse intramural esophageal pseudodiverticulosis. New cases and review. Gastroenterology. 1977 Mar;72(3):541-5.

6 Medeiros LJ, Doos WG, Balogh K. Esophageal intramural pseudodiverticulosis. A report of two cases with analysis of similar, less extensive changes in "normal" autopsy esophagi. Hum Pathol. 1988 Aug;19(8):928-31.

7 Kataoka H, Higa T, Koono M. An autopsy case report of diffuse esophageal intramural pseudodiverticulosis. Acta Pathol Jpn. 1992 Nov;42(11):837-40.

8 Shintaku M, Nishida T, Shiomi K, Shintaku M. Active opening and closing movements of the orifices of esophageal intramural pseudodiverticulosis. Gastrointest Endosc. 2011 Dec;74(6):1420-2.

9 Hentschel F, Lüth S. Clinical and endoscopic characteristics of diffuse esophageal intramural pseudo-diverticulosis. Esophagus. 2020 Oct;17(4):492-501.

10 Nakanishi Y, Ochiai A, Shimoda T, Yamaguchi H, Tachimori Y, Kato H, et al. Epidermization in the esophageal mucosa. Unusual epithelial changes clearly detected by Lugol's staining. Am J Surg Pathol. 1997 May;21(5): $605-9$.

11 Ezoe Y, Fujii S, Muto M, Ochiai A, Ohtsu A. Epidermoid metaplasia of the esophagus. Endoscopic feature and differential diagnosis. Hepatogastroenterology. 2011 MayJun;58(107-108):809-13.

12 Taggart MW, Rashid A, Ross WA, Abraham SC. Oesophageal hyperkeratosis. Clinicopathological associations. Histopathology. 2013 Oct;63(4):463-73.

13 Singhi AD, Arnold CA, Crowder CD, Lam-Himlin DM, Voltaggio L, Montgomery EA. Esophageal leukoplakia or epidermoid metaplasia. A clinicopathological study of 18 patients. Mod Pathol. 2014 Jan;27(1):38-43.

14 Bertoni G, Sassatelli R, Nigrisoli E, Conigliaro R, Bedogni G. Ectopic sebaceous glands in the esophagus. Report of three new cases and review of the literature. Am J Gastroenterol. 1994 Oct;89(10):1884-7.

15 Long JD, Orlando RC. Esophageal submucosal glands. Structure and function. Am J Gastroenterol. 1999 Oct; 94(10):2818-24. 